\title{
CONCENTRATION-TIME COURSE IN THE PLASMA OF MAN OF RADIOMERCURY INTRODUCED AS A MERCURIAL DIURETIC ${ }^{1}$
}

\author{
By S. A. THREEFOOT, C. T. RAY, G. E. BURCH, J. A. CRONVICH, ${ }^{2}$ \\ J. P. MILNOR, ${ }^{3}$ W. OVERMAN, AND W. GORDON \\ (From the Department of Medicine, Tulane University School of Medicine, and Charity \\ Hospital of Louisiana, Nerw Orleans)
}

(Received for publication December 20, 1948)

\section{INTRODUCTION}

During the course of investigations into the mechanism of congestive heart failure and other states of edema, it became evident that there was need for further understanding of the pharmacodynamics of the mercurial diuretics, including their distribution, mechanism of action, and elimination. Studies based upon chemical methods of analysis are limited because of the minute concentrations of the mercurial compounds present in the body fluids and tissues and the number of samples which can be analyzed in a given time. The use of the tracer technic made possible the extension of these studies to smaller quantities and to a greater number of determinations.

This presentation is directed at a better definition of the concentration-time course of mercury intravenously injected as a diuretic into the circulating plasma of man. A mercurial diuretic (Mercuhydrin ${ }^{4}$ ) labeled with radioactive mercury ${ }^{5}$ was employed in the tracer studies. It is well to realize that it is the mercury which is being traced and not the molecule of the diuretic.

1 Aided by grants from the Life Insurance Medical Research Fund, a War Department Grant No. W-49007-MD389, and the Mrs. E. J. Caire Fund for Research in Heart Disease.

2 Department of Medicine and School of Electrical Engineering, Tulane University of Louisiana.

3 National Institute of Health Post-doctorate Research Fellow, Department of Medicine, Tulane University School of Medicine, and Charity Hospital of Louisiana at New Orleans.

4 The sodium salt of methoxyoximercuripropylsuccinylurea with theophylline, prepared with radiomercury in this laboratory by Messrs. Harold Krahnke, Darwin Kaestner and Edwin Sprengler through the courtesy of Dr. H. L. Daiell, Director of Research, of Lakeside Laboratories, Milwaukee.

5 Radioactive mercury $\left(\mathrm{Hg}^{203,205}\right)$ was obtained from the Isotopes Division of the Oak Ridge National Laboratories.

\section{MATERIALS AND METHODS}

Fifteen subjects were studied. All except one were patients on the medical wards at the Charity Hospital. Certain clinical information concerning these subjects is presented in Table I.

The subjects were permitted to rest comfortably in bed in an air-conditioned room for 30 to 60 minutes in order to attain a stable state. Whenever the subject's clinical condition permitted, samples of urine were collected by means of a bladder catheter.

Two cc. of the radioactive mercurial diuretic, containing $78 \mathrm{mg}$. of total mercury, was injected intravenously over a period of about 20 seconds. Collection of blood samples was begun simultaneously with the beginning of the injection. Two types of collections were made: 1 . In some subjects blood was withdrawn, by means of a syringe and a heparinized 20-gauge needle, from an antecubital vein contralateral to that into which the injection had been made. For the first two to three minutes, samples were collected as rapidly as 2 cc. of blood could be withdrawn (approximately four to eight seconds). The interval between collections was progressively increased every five to 10 minutes until at the end of one hour, collections were being made every 15 to 30 minutes for the remainder of the study, which lasted 100 to $180 \mathrm{~min}-$ utes in all. 2. In another group of subjects, blood was taken from a femoral artery. It was allowed to flow freely from the open needle for the first two to three minutes, the time between collection of 2 cc. samples varying from two to five seconds. This interval was then progressively lengthened as described for the venous collections.

The blood samples were centrifuged and $0.3 \mathrm{cc}$. of the serum was dropped upon a filter paper disc, allowed to dry and cemented to a metal disc for counting.

Urine was collected as rapidly as samples of sufficient quantity for counting could be obtained. There was usually an interval of five to 15 seconds between collections. After the period of rapid collections of the blood samples, a sample of urine was obtained as nearly coincident as possible with the time of each blood sample.

Radioactive measurements were made with thin micawindow Geiger-Müller counter tubes or a continuous gas-flow counter tube. All measurements were recorded as counts per minute per cubic centimeter of fluid and were corrected to values corresponding to an injected dose of radiomercury with an activity of $10,000,000$ counts per minute. 
TABLE I

Summary of clinical data of the 15 patients studied

\begin{tabular}{|c|c|c|c|c|c|c|c|}
\hline Subject & Age & Sex & Weight & Diagnosis & Status & $\begin{array}{l}\text { Severity } \\
\text { of edema }\end{array}$ & $\begin{array}{l}\text { Previous } \\
\text { mercurial } \\
\text { diuretic }\end{array}$ \\
\hline $\begin{array}{l}1 \\
2\end{array}$ & $\begin{array}{l}58 \\
40\end{array}$ & $\begin{array}{l}\mathrm{M} \text {. } \\
\mathrm{F}\end{array}$ & $1 \overline{53}$ & $\begin{array}{l}\text { Cirrhosis with ascites } \\
\text { Hypertensive cardiovascular disease, } \\
\text { congestive heart failure }\end{array}$ & $\begin{array}{l}\text { Stationary } \\
\text { Stationary }\end{array}$ & $\begin{array}{l}2+ \\
3+\end{array}$ & $\begin{array}{l}\text { None } \\
\text { Four } \\
\text { days } \\
\text { prior }\end{array}$ \\
\hline 3 & 64 & M. & 160 & $\begin{array}{l}\text { Hypertensive cardiovascular disease, } \\
\text { congestive heart failure }\end{array}$ & Improving & $3+$ & None \\
\hline 4 & 59 & F. & 139 & $\begin{array}{l}\text { Hypertensive and syphilitic cardiovas- } \\
\text { cular disease; congestive heart failure }\end{array}$ & $\begin{array}{l}\text { Worse on admission; } \\
\text { improved after } \\
\text { mercurial diuretic }\end{array}$ & $4+$ & $\begin{array}{l}\text { None } \\
\text { day } \\
\text { before }\end{array}$ \\
\hline 5 & 53 & M. & 140 & $\begin{array}{l}\text { Syphilitic heart disease, congestive } \\
\text { heart failure }\end{array}$ & Improving & $0-1+$ & None \\
\hline 6 & 58 & M. & 154.5 & $\begin{array}{l}\text { Arteriosclerotic heart disease, con- } \\
\text { gestive heart failure }\end{array}$ & Rapidly improving & $3+$ & None \\
\hline 7 & 34 & M. & 120 & $\begin{array}{l}\text { Congenital heart disease; unilateral } \\
\text { lymphedema, right leg }\end{array}$ & $\begin{array}{l}\text { No failure; } \\
\text { stationary }\end{array}$ & $\begin{array}{c}4+ \\
\text { right leg }\end{array}$ & None \\
\hline $\begin{array}{l}8 \\
9\end{array}$ & $\begin{array}{l}21 \\
33\end{array}$ & F. & $\begin{array}{r}92 \\
155\end{array}$ & $\begin{array}{l}\text { Cervical lymphadenitis } \\
\text { Pulmonary abscess }\end{array}$ & $\begin{array}{l}\text { Improving } \\
\text { Recovered }\end{array}$ & $\begin{array}{l}0 \\
0\end{array}$ & None \\
\hline 10 & 60 & $\mathrm{~F}$. & 121.5 & Diabetes & Controlled & 0 & $\begin{array}{l}\text { None } \\
\text { None }\end{array}$ \\
\hline 11 & 34 & M. & 176 & Chronic malaria & Recovered & 0 & None \\
\hline 12 & 21 & $\mathrm{~F}$ & 106.5 & Postpneumonia & Recovered & 0 & None \\
\hline 13 & 61 & M. & 135.5 & Lymphoma & Stationary & 0 & None \\
\hline 14 & 32 & M. & 126.5 & Peptic ulcer & Recovered & 0 & None \\
\hline 15 & 34 & M. & 156.5 & Amebic colitis & Improving & 0 & None \\
\hline
\end{tabular}

\section{DATA AND METHODS OF ANALYSIS}

Since the experimentally observed values of this study could not be made to fit precisely any regularly shaped curve, it may be considered better to abandon the idea of ever being able to define the regression of mercury from the blood until the methods of biologic analysis have been considerably improved. To take this course would undoubtedly remove criticism from the analyses and would eliminate the development of erroneous concepts. However, there would be little purpose in the mere presentation of observed values of blood concentration for radiomercury. Furthermore, if the nature and significance of the mathematical analyses of the data observed are properly understood, and if it is also recognized that such analyses extremely oversimplify the physicochemical processes related to the regression in concentration of mercury, so that they represent only an attempt to approach the definition of these complex processes, no misconceptions should result and discussions should follow. The latter point of view has been accepted for the presentation of these data, and discussions will include mathematical expressions which are only approximations of the biologic processes involved in the observed data.

The mathematical approach to the analysis of blood regression curves of radiosodium in dogs and in guinea pigs has been presented in detail by Gellhorn and associates (1), and in man by workers in this laboratory (2). Again, this method has been employed as an arbitrary approach to the analysis of the concentration-time course of radiomercury in man. The observed values were plotted on semilogarithmic paper and a close-fitting curve (frequently referred to below as the "mother" curve) was drawn in by inspection (Figure 1). The general equa- tion for such a curve is:

$$
C_{p}=A_{1} e^{-b_{1} t}+A_{2} e^{-b_{2} t}+A_{3} e^{-b_{3} t}+\cdots A_{n} e^{-b_{n} t},
$$

where $C_{p}$ is the concentration of radiomercury in the serum at time $(t)$.

$A_{1}, A_{2}, A_{3}, \cdots A_{n}=$ the respective amounts of the tracer which are regressing at the different rates.

$b_{1}, b_{2}, b_{3}, \cdots b_{n}=$ the rates of movement per minute of the respective amounts of the tracer out of the serum. The absolute values of these symbols indicate the portions of $A_{1}, A_{2}, \cdots A_{n}$, respectively, which leave the serum per unit of time.

There are numerous rates of movement or regression contributing to the observed curves, but because of the nature of the data and the curves, it was considered impractical to carry the analysis into more than three rates. During the interval of these studies the concentration of radiomercury in the serum continued to decline but never reached a constant value; therefore, the absolute value of $b_{3}$ in the third member of the equation was neither zero nor small enough to be considered as zero. This differs from the regression of sodium in that the concentration of the latter approached a constant value more closely. In fact, in the sodium studies such a value was considered for convenience to exist and, therefore, the $C_{\text {eq }}$ value was employed, or $b_{3}$ was considered to equal zero (1).

The portion of the equation represented by the expression $A_{3} e^{-b_{3} t}$ was the tangent drawn through those points which most closely approached a horizontal and straight line in the "mother" curve (Figure 1). Selected values along this line extending to the ordinate $(t=0)$ were subtracted from simultaneously occurring values along the "mother" curve. These differences were plotted, and a curve was drawn through the points. The expression of 
the tangent to this new curve through the points which most closely approached a straight line was represented by $A_{2} e^{-b_{2} t}$. Selected values along this line extending to the ordinate were subtracted from simultaneously occurring values of the remainder of the curve not tangent to the line represented by $A_{2} e^{-b_{2} t}$; the differences were again plotted and a line was drawn through these points. The expression of this final "daughter" line, or regression rate, is represented by $A_{1} e^{-b_{1} t}$. It is evident from Figure 1 that it would have been impractical to carry the analysis further. Therefore, there appear to be at least three major rates of regression, each probably representing many physicochemical processes mathematically integrated to define the declination in concentration of mercury in the plasma of man. The sum of the values along these "daughter" curves at any time $(t)$ represents the total concentration of the isotope in the plasma at that time, expressed graphically by Figure 1 or by equation (1). A discussion of the significance of these various regression rates will follow.

When the observed values from about two to about 200 minutes are plotted on logarithmic coordinates, they tend to lie along a straight line, the equation for which is $C_{p}=A t^{-b}$, where

$C_{p}=$ concentration of radioactive mercury in plasma at any time $(t)$
$A=$ a constant which is numerically equal to the concentration of radioactive mercury in the plasma at one minute

$t=$ time in minutes after injection

$b=$ ratio of percentage change in concentration to percentage change in time (the slope of the curve on logarithmic coordinates).

Figure 2 permits the comparison of the curves of observed values for a subject plotted on recti-. linear, semilogarithmic and logarithmic coordinates.

The regression curves of five of the 15 subjects studied were chosen for critical analysis. In these five the specific radioactivity of the plasma was sufficiently great to insure a high degree of accuracy in counting throughout the period of study. In Figure 3 these values are plotted on rectilinear (lower curves) and logarithmic coordinate (upper curves) systems. The regression curves or rates of regression of mercury from the plasma were similar for all 15 patients. Figure 4 illustrates a composite curve of all patients plotted on the semilogarithmic coordinate system. The degree of the

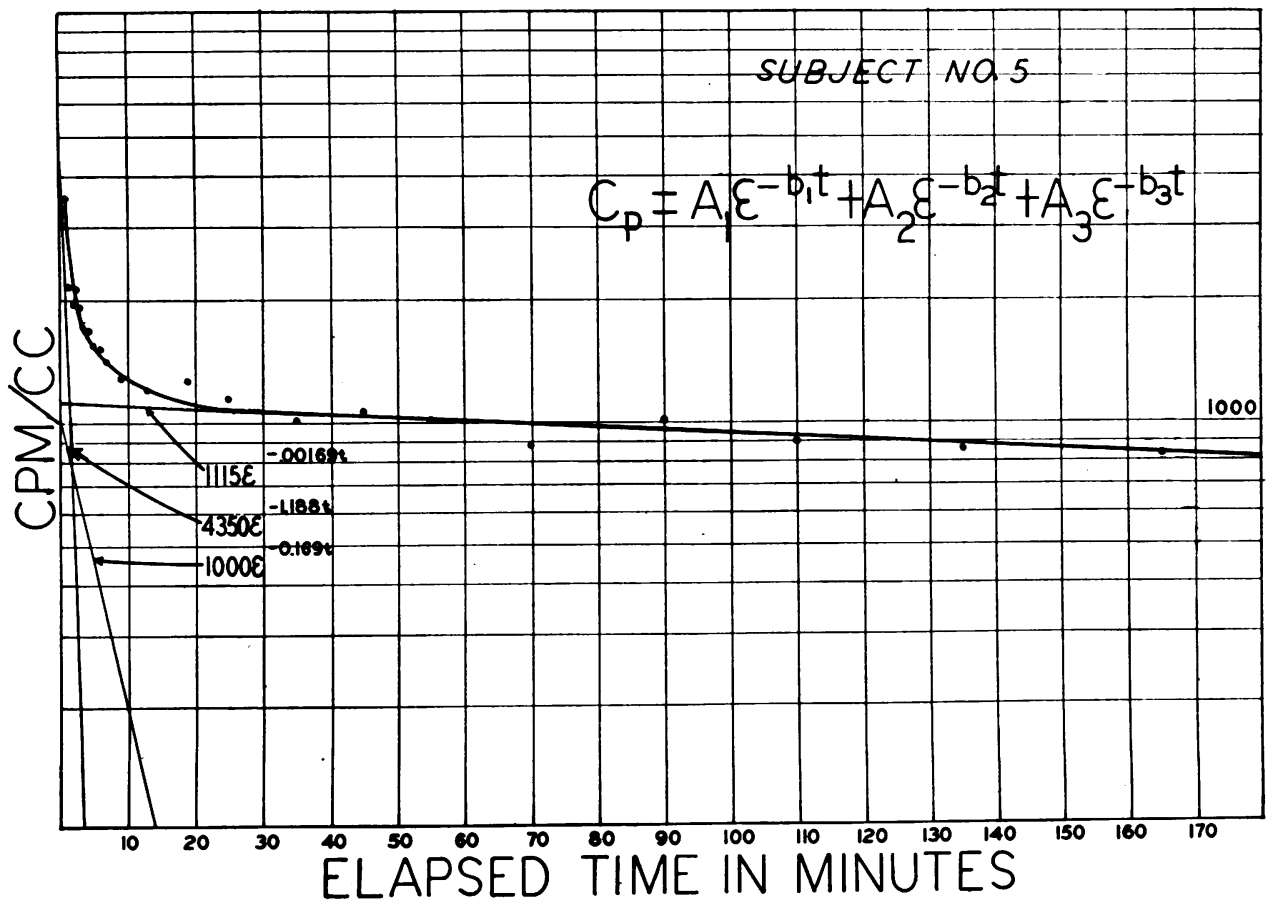

Fig. 1. The Observed Points and Mean Curve of the Concentration-Time Course of Radiomercury in the Plasma of Subject 5

Three regression rates are shown and defined by the equation $C_{p}=4350 e^{-1.188 t}+1000 c^{-0.169 t}$ $+1115 e^{-0.00169 t}$. 

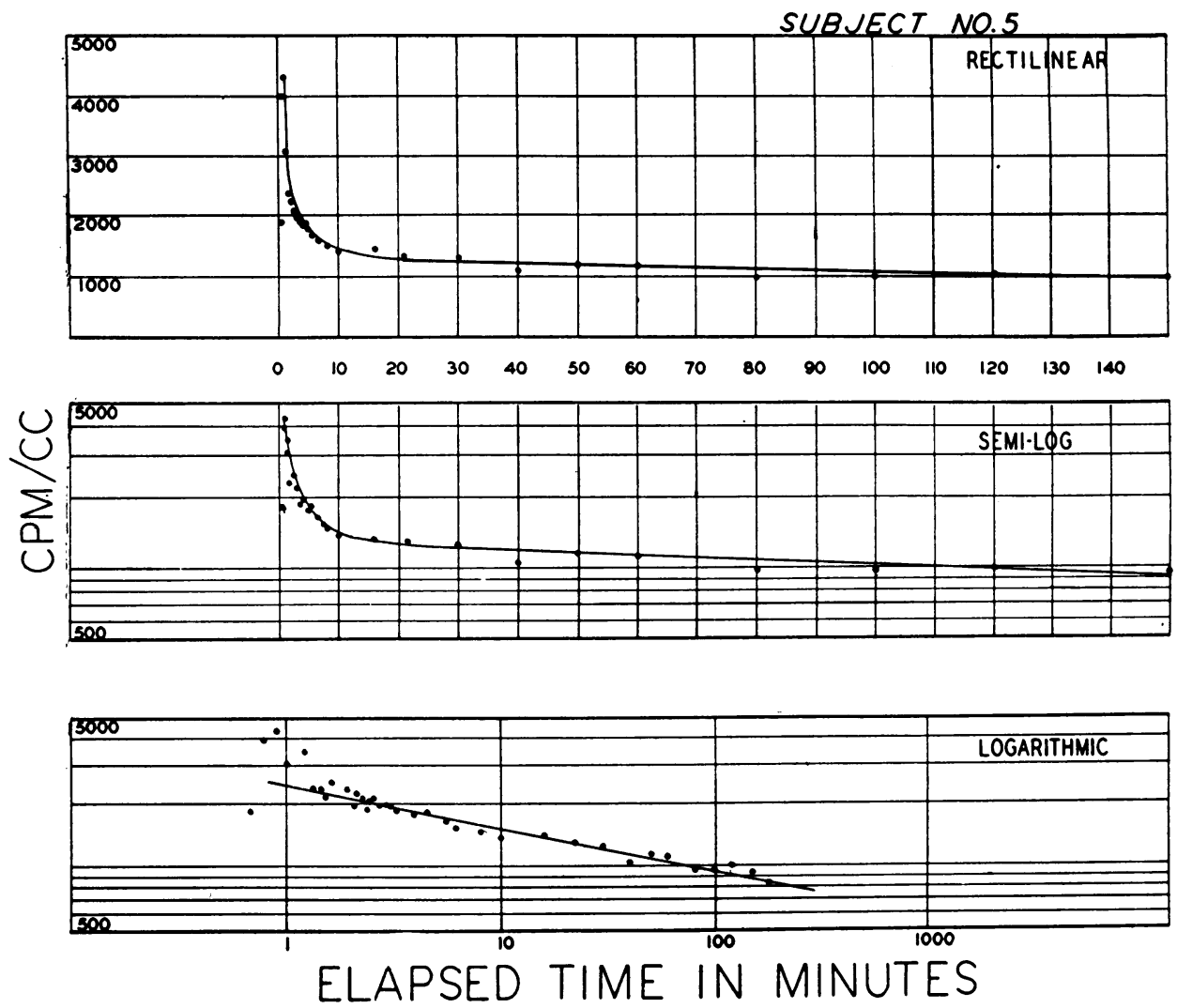

Fig. 2. Concentration-Time Course of Radiomercury in the Plasma of Subject 5 Plotted on Three Different Coordinate Systems

Note that a considerable number of the observed points tend to lie along a straight line drawn on the logarithmic coordinate system.

individual variations precluded a differentiation of the curves or rates of regression of one clinical state from that of another, since small differences would have no statistical significance in such a limited series.

Figure 5 illustrates the regression curve obtained in similar experiments with the use of $\mathrm{Na}^{22}$ as a tracer. ${ }^{6}$ The regression curves of one of the studies with radioactive mercury and one with radiosodium plotted on logarithmic coordinate system permit comparison of the concentrationtime course of these elements in the plasma of man. The differences are self-evident, especially those existing after approximately 200 minutes.

6 These studies were conducted primarily for other purposes at Grady Hospital, Emory University Medical School, Atlanta, in collaboration with Drs. A. J. Merrill and Heywood Turner. The samples were collected in Atlanta and brought to New Orleans for counting.

\section{DISCUSSION}

The interpretation of the regression curves obtained for any tracer is complicated by the combination effects of simultaneously occurring phenomena. This is particularly true in the initial portion of the curves, since all processes concerned, rapid and slow, are manifested at this time, when the greatest changes in concentration are also taking place. Progressively the tracer recording those factors responsible for rates of rapid change approaches equilibrium level and manifests itself less and less in the completed regression curve.

As has been demonstrated previously for sodium (2), and in these studies for mercury, there are essentially three separate major regression rates which contribute to the decline in concentration of the tracer in the serum. Obviously, the initial rate of regression of the concentration of radio- 


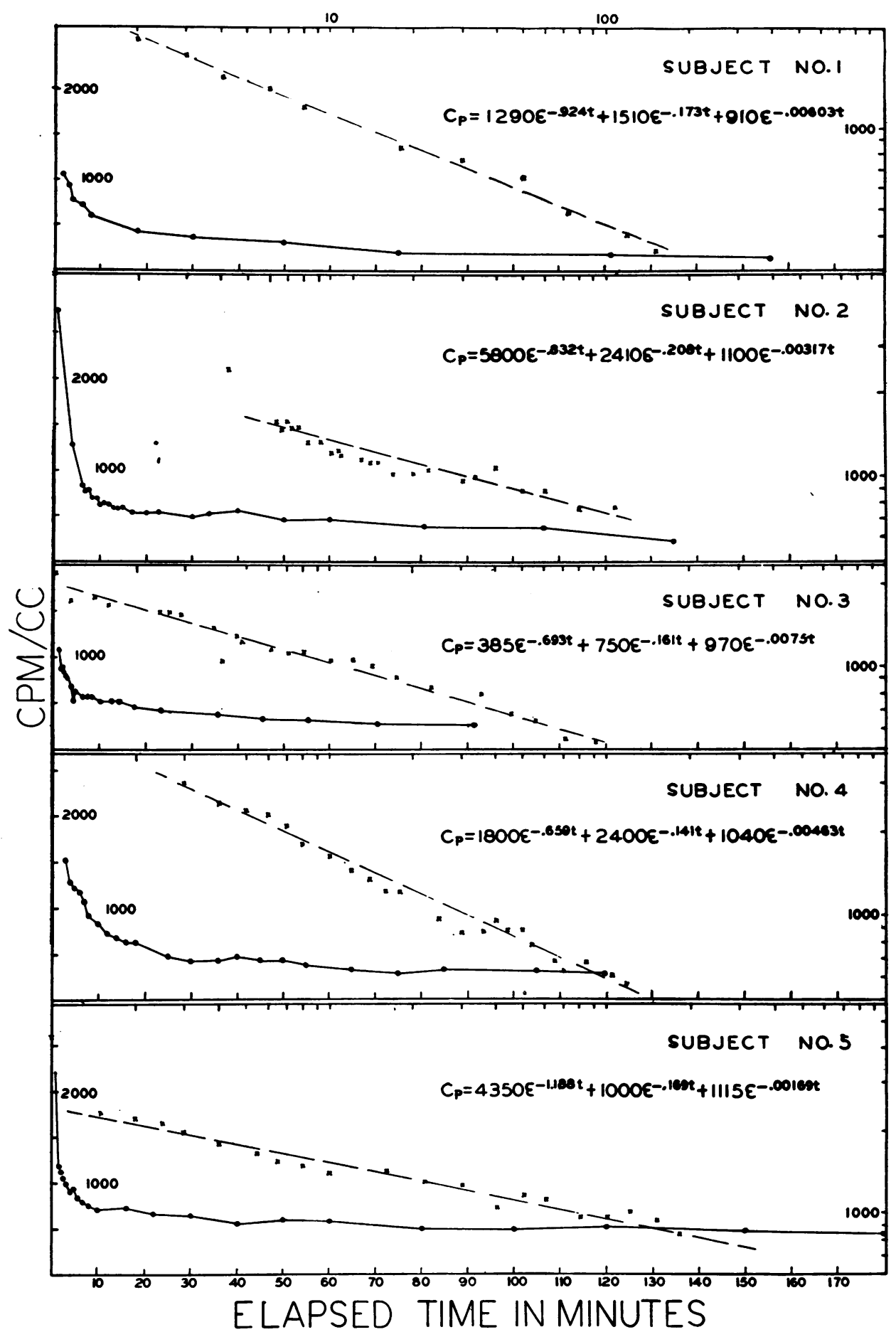

Fig. 3. The Concentration-Time Course of Radiomercury in the Plasma of Subjects 1-5 Plotted on Both Rectilinear and Logarithmic Coordinates

The coordinates for the logarithmic graph are on the top and the right of each graph and for the rectilinear on the left and bottom. The equations shown were obtained by the semilogarithmic graphic method of analysis described in the text. 


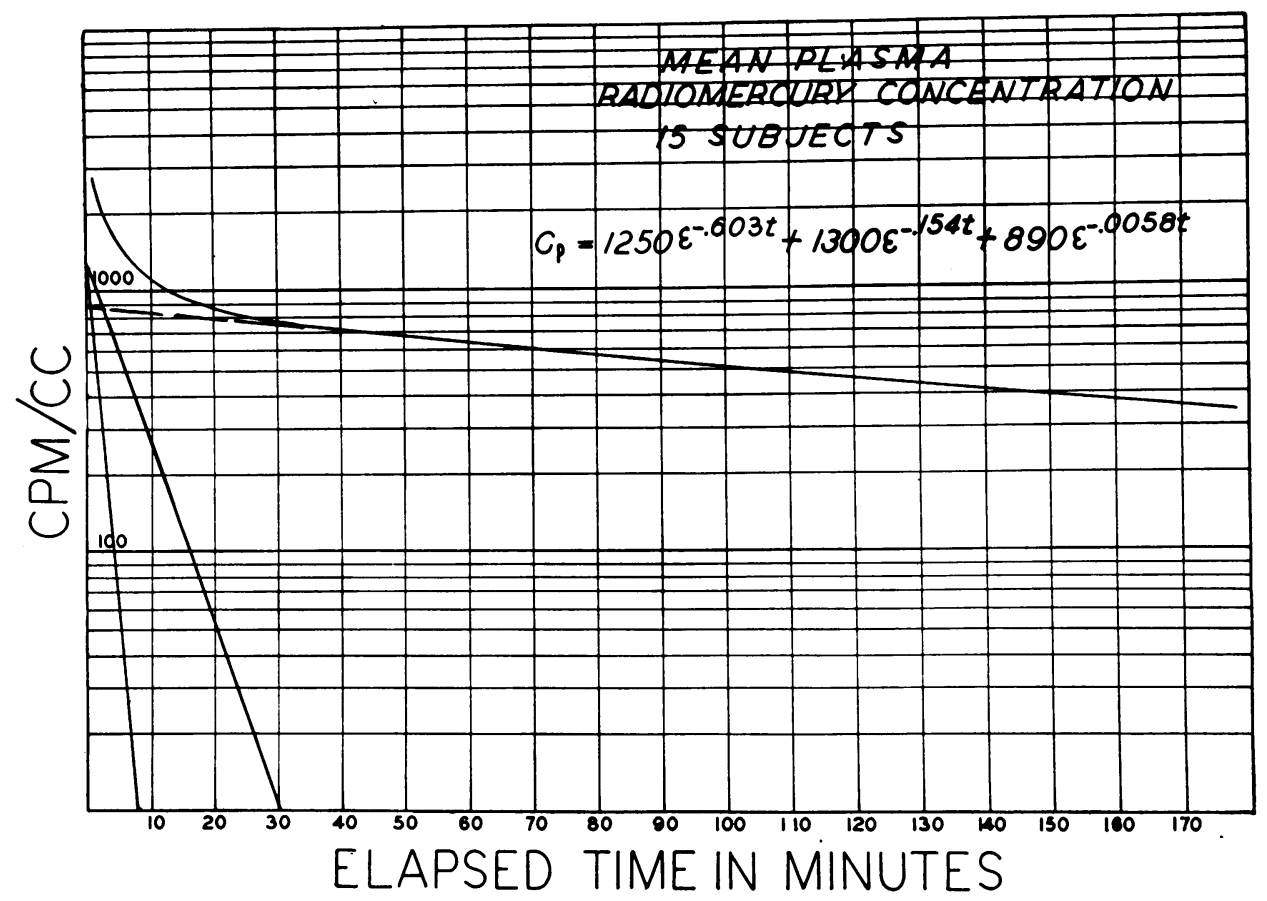

Fig. 4. The Mean Observed Curve and Equation of Cencentration-Time Course of Radiomercury in the Plasma of 15 Subjects

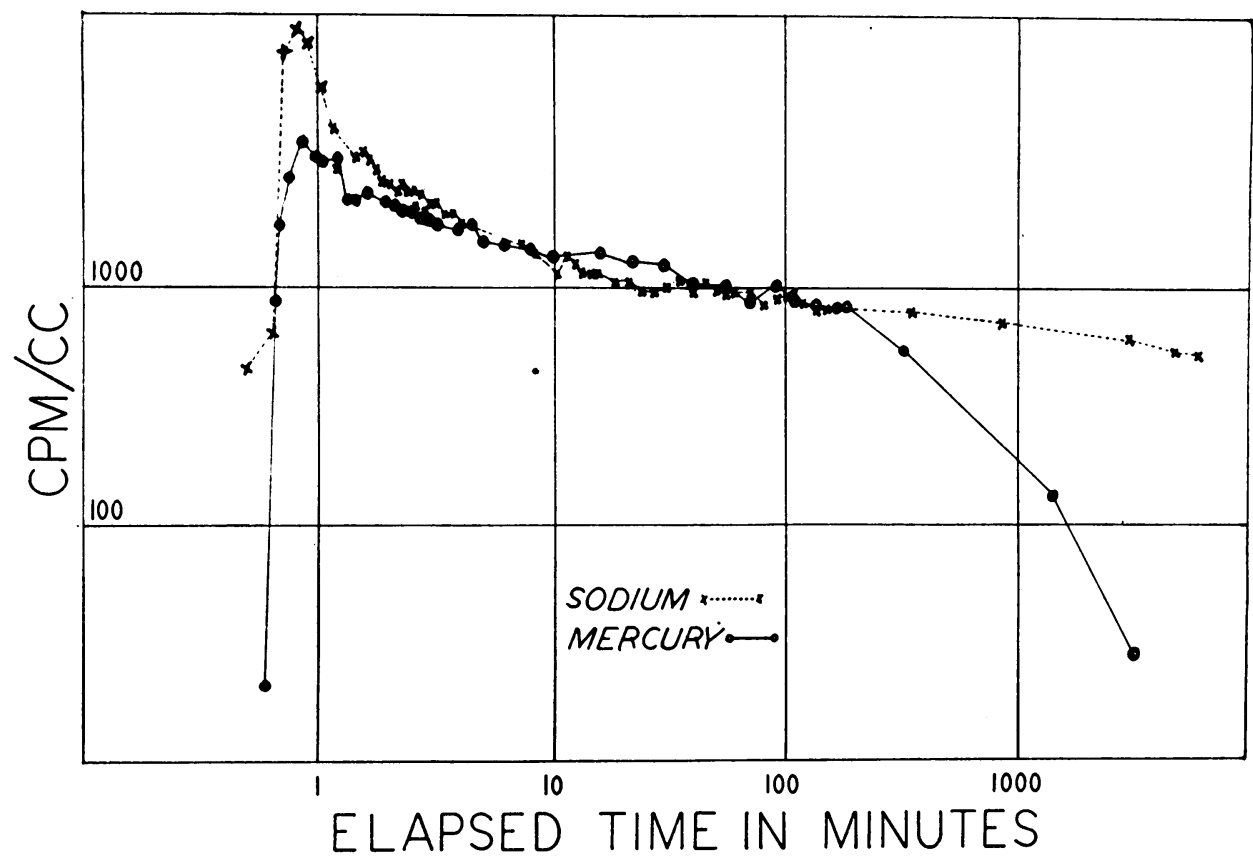

Fig. 5. The Plasma Concentration-Time Course of Radiosodium Compared with that of Radiomercury in Two Different Subjects Plotted on Logarithmic Coordinate System

Note that whereas the curves are similar, they differ in the early portion, and that after 200 minutes the curves separate, the concentration of the mercury declining rapidly, with that of sodium being maintained. The ordinate values for both mercury and sodium were rendered comparable. 
mercury in the plasma is influenced by the phenomenon of mechanical mixing. Figure 6 illustrates the ascension of concentration as well as the early portion of regression. A column of blood passing through the antecubital vein during the period of injection will contain a high concentration of radiomercury. The local variations in concentration within the column will vary with the rate (force or linear rate and volume) of delivery of the radioactive substance. Since the collection of the blood samples begins simultaneously with the injection of the labeled substance, the first few samples collected will contain no radiomercury, the time of appearance of the tracer being dependent upon the circulation time and the rate and force of injection. When the tracer-containing blood column passes through the right side of the heart, its specific radioactivity is reduced by dilution with blood returning from other parts of the body; in the pulmonary vessels vigorous mixing takes place; and upon entering the left chambers of the heart, the radioactive column undergoes further mixing. Several ventricular contractions, at least as many as occurred during the time required for injection of the radiomercury into the antecubital vein, are required to force the greater portion of the radioactive column of blood into the systemic circulation. From the moment the left ventricle receives radioactive blood, all blood leaving it will be radioactive because:

1. The column of blood into which the injection was made will contaminate the walls of all vessels through which it flows, which in turn contaminate in-flowing nonradioactive blood.

2. The blood which contains radioactive mercury ejected from the left ventricle will flow through vascular circuits of variable length, returning from the shortest (coronary circulation) before the return from the longest (lower extremity) has begun. This effects rapid, complete mechanical mixing and early, continuous return of radioactive blood to the left ventricle.

3. The linear velocity of blood flow through a vessel decreases from the center of the lumen of the vessel to the wall, the flow being almost zero at the surface of the wall. This phenomenon continuously increases the length of the column of radioactive blood, so that a continued flow of radioactive blood into the heart is maintained and mechanical mixing is encouraged. The process of axial flow and surface skimming in vessels other than the segment receiving the injection will tend to delay mechanical mixing.

Under ideal experimental conditions the tracer substance should be completely mixed and distributed homogenously in the blood at the instant of injection. A concentration-time curve would then be concerned with biologic phenomena other than mixing. Obviously, this ideal experimental condition cannot be achieved. An alternative approach might consist in delaying observations until mixing was completed; this, however, would be unsatisfactory because the movement of mercury into some compartments might be so rapid that the mercury in the blood might reach equilibrium with that in these compartments during the period allowed for mixing and before the observations are begun. The physiologic data available today strongly suggest that mixing has very little or no influence upon the regression curve after $30 \mathrm{~min}$ utes, probably negligible influence after 15 minutes in all subjects and even after one or two minutes in some subjects.

It becomes evident, therefore, that with rapid loss of a tracer substance from the blood and with relatively slow mechanical mixing, it is impossible to obtain an absolute picture of the true rate of loss of a tracer from the blood stream of man. It is also apparent that a true picture cannot be obtained from the later periods alone of the regression curve, because the tracer method is dependent upon the principle that all turnover phenomena, regardless of number and rate, are occurring at all times of study, special physiologic situations excluded.

It is safe to state that the initial portion of the concentration-time curve is the resultant of all the regression forces acting on mercury in the plasma stream of man and that the initial regression rate expressed as $b_{1}$ (Figures 1 and 4 ) is a manifestation largely of mixing processes occurring simultaneously with other rapid biologic phenomena of a physicochemical nature, such as fixation to protein molecules and cell surfaces. The other rates of regression are probably so little influenced by mixing that this phenomenon may be eliminated from serious consideration except insofar as mixing or continued circulation of the blood and other body fluids is necessary to insure satisfactory continuation of the other physicochemical processes 
more directly responsible for the concentrationtime course of mercury in the plasma.

The second regression rate is dependent upon those factors which are more apparent after the mixing process has been completed, for example, slower rates of diffusion into tissue spaces, cells, bone, and other sites of the body. Some of these have been discussed for sodium (3) and will not be repeated in detail.

It is well to realize that when $\mathrm{Na}^{24}$ or $\mathrm{Na}^{22}$ is employed as a tracer and is added to an organism such as man, it labels an already-existing, dynamic state of turnover of sodium among the various compartments within the body, i.e., in the normal subject, except under special circumstances, the sodium space is already saturated with relatively large quantities of nontracer sodium. Therefore, the various components of the regression curves of tracer sodium represent its movement into sodium compartments already in a steady state concerned with active processes in dynamic equilibrium. Mercury, on the other hand, does not exist within the body under comparable conditions. That which is present normally in the body is apparently a contaminant and exists in relatively small quantities (4). It is possible, however, that the "saturated" state for mercury in the body is zero concentration and that the body mechanisms are set to maintain this level. Nevertheless, there exists essentially a "turnover pattern" for mercury, which, unlike that for sodium, is not active. These innumerable turnover rates or regression processes for mercury manifest themselves only when the element is present, that which is normally present as a contaminant being disregarded. Thus, the radioactive mercury injected as an extremely small part of a total of $78 \mathrm{mg}$. of mercury in the mercurial diuretic labels practically all of the mercury which exists in the body (4) and which activates the "turnover patterns" for mercury. The regression curves of the radioactive mercury then must represent partially the migration of mercury into "depots" or "relatively fixed" compartments to fill "potential mercurial spaces." The result is an essentially unidirectional loss of mercury from the circulating serum rather than an exchange process already in equilibrium. The compartments into which mercury moves most probably include those systems concerned with the protection of the body from toxic effects of this foreign substance and with its subsequent excretion, for example, the liver, kidneys and cells and

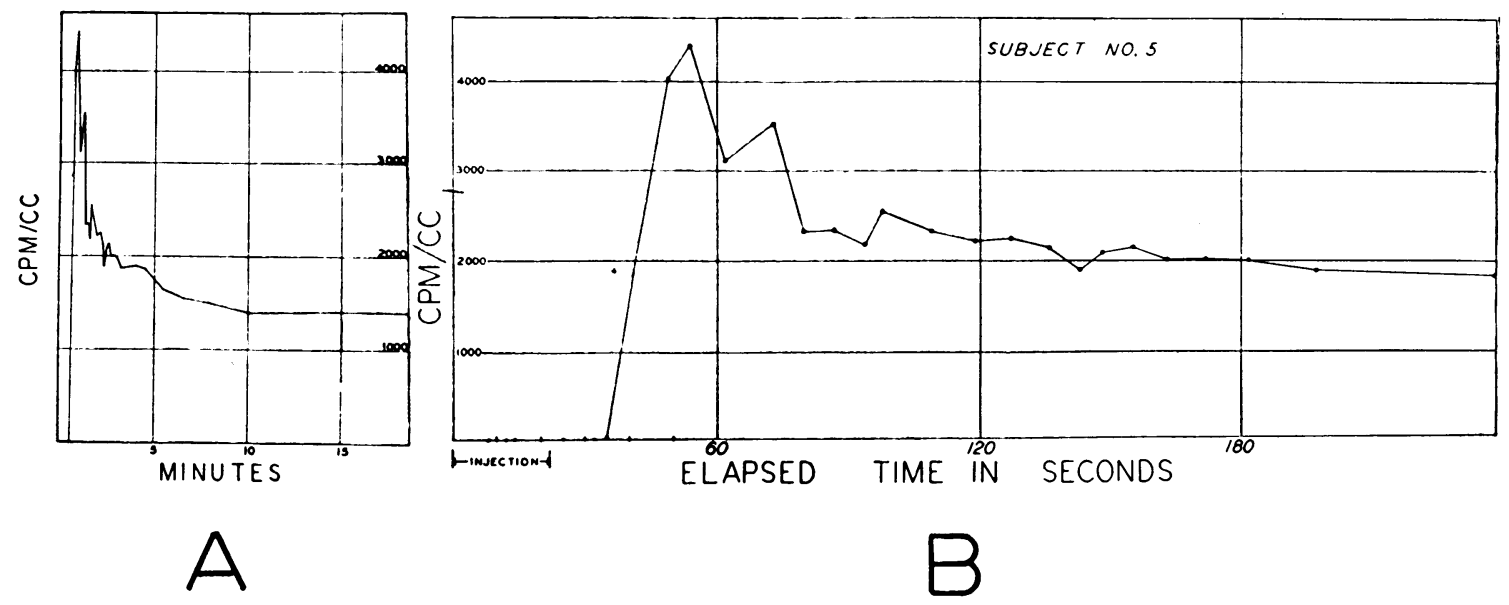

FIG. 6

A. The ascension and regression of the concentration of radiomercury in the plasma for the first 20 minutes after injection.

B. The first four minutes of curve A more clearly illustrate the early variations in concentration. Note that radioactivity first appeared in the blood of the femoral artery between 35 and 40 seconds after the beginning of the injection into an antecubital vein. Twenty-two seconds were required for the injection.

The intersection (extrapolated) on the ordinate at 2,800 c.p.m. indicates the concentration if mixing were complete at $t_{0}$. Except for errors due to the loss of mercury from the plasma during the first three minutes, the ratio of the injected dose to this extrapolated concentration "indicates" the mercurial space which at $t_{0}$ is also the plasma volume, since mercury does not adhere to erythrocytes in significant amounts. 


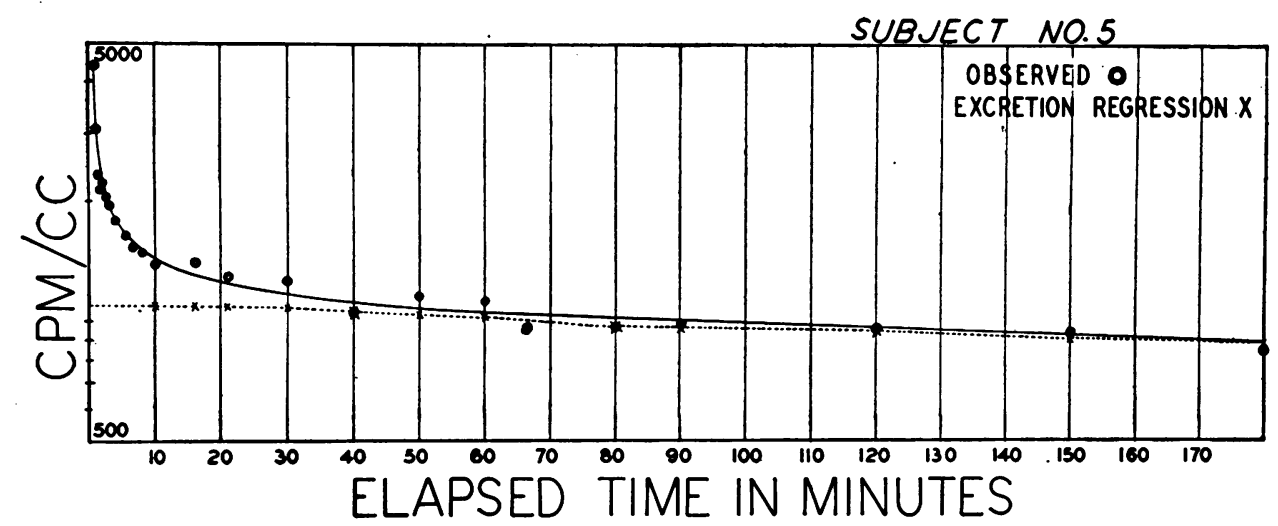

FIG. 7

The interrupted line indicates theoretic concentration of radiomercury in the plasma calculated from that excreted in the urine and compared with the observed concentration of radiomercury in the plasma.

protein compartments not in the blood stream. It is likely that the recorded regression phenomena for mercury would be entirely different if the mercury depots were already saturated and the turnover processes were progressing at full pace instead of existing merely as "potential turnover patterns."

After the relatively rapid phenomena have manifested themselves in the first two regression rates, the relatively slow excretory rate is more clearly observed as a significant part of the third regression rate (Figures 1 and 7 ). That this third rate of decline in concentration of the radioactive mercury in the serum is a reflection primarily of urinary excretion is supported by the fact that the urinary excretion appears to account for a considerable part of the loss of radiomercury from the serum of some subjects beyond approximately 60 minutes after the time of injection. In other subjects the urinary excretion may not account for as much of the decline but is responsible for a substantial fraction of the regression from the blood. Details of urinary excretion will be presented elsewhere. Other routes of excretion undoubtedly influence this portion of the curve also.

Since the segment of the curve of the concentration-time course of radiomercury in the interval from about two to 200 minutes appears to be almost linear on logarithmic coordinates (Figures 2 and 3 ), the ratio of the percentage change in concentration to percentage change in time is nearly constant in this interval. The significance of this has not been fully developed.
It should be pointed out that the differences in the regression curves of sodium and mercury (Figure 5) are probably due to the differences in the respective phenomena which they represent. The rate of regression of sodium is more rapid early and considerably less rapid later. This is expected, since sodium diffuses more readily but is excreted more slowly than mercury.

The relationships of concentrations of radioactive mercury in blood serum to those in other biologic fluids, including urine, are under study at the present time and will be discussed elsewhere. With better understanding of the nature of the regression of mercury from the plasma, other biologic studies employing mercury in man can be better planned and evaluated. Without detailed knowledge of the concentration-time course of mercury in the blood plasma, accurate experiments concerned with blood values would be impossible.

\section{SUM M ARY}

The concentration-time course of a mercurial diuretic labeled with radioactive mercury and injected intravenously has been studied in 15 human subjects. Analysis of the regression curves demonstrates that there are at least three major exponential rates responsible for the decline in concentration of mercury in the plasma. The first rate is apparently dependent to a large extent upon mechanical mixing; the second is possibly due to relatively rapid adsorptive, chemical and diffusion phenomena with migration into dynamic and more stable compartments; the third rate is 
most probably a reflection mainly of excretory processes, principally urinary. It was not possible from these data to draw any conclusions as to differences because of the various clinical states in the subjects studied. Comparison of these curves with those similarly obtained for sodium indicate that there may be slight differences in the early portions of the regression curves and that great differences exist in those portions of the curves dependent upon the excretion of the isotope. The concentration of sodium in the plasma practically reaches an equilibrium, whereas the concentration of mercury may reach a steady state but does not approach an equilibrium due, in part, to rapid elimination.

These observations make possible a better understanding of the pharmacodynamics of mercurial diuretics and certain physiologic phenomena and thus facilitate better planning of experimental studies concerned with mercurial diuretics necessarily based upon plasma concentration of mercury.

\section{BIBLIOGRAPHY}

1. Gellhorn, A., Merrell, M., and Rankin, R. M., Rate of transcapillary exchange of sodium in normal and shocked dogs. Am. J. Physiol. (Oct.), 1944, 142, 407.

2. Burch, G., Reaser, P., and Cronvich, J., Rates of sodium turnover in normal subjects and in patients with congestive heart failure. J. Lab. \& Clin. Med. (Oct.), 1947, 32, 1169.

3. Merrell, M., Gellhorn, A., and Flexner, L. B., Studies on rates of exchange of substances between blood and extravascular fluid; exchange of sodium in the guinea pig. J. Biol. Chem., 1944, 153, 83.

4. Sollmann, T., A Manual of Pharmacology. W. B. Saunders Co., Philadelphia, 1942, Ed. 6. 